\title{
Effects of Healthy Aging on Right Ventricular Structure and Biomechanical Properties
}

\author{
Short Title: Effects of Aging on RV Biomechanics
}

Danial Sharifi Kia, $\mathrm{MSc}^{1}$, Yuanjun Shen, $\mathrm{PhD}^{2}$, Timothy N. Bachman, $\mathrm{MSc}^{1,2}$, Elena A.

Goncharova, $\mathrm{PhD}^{1,2,3,4}$, Kang Kim, $\mathrm{PhD}^{1,2,3,5,6,7,8}$, Marc A. Simon, MD, MSc ${ }^{1,2,3,5,6^{*}}$

${ }^{1}$ Department of Bioengineering, University of Pittsburgh, Pittsburgh, PA

${ }^{2}$ Pittsburgh Heart, Lung, Blood and Vascular Medicine Institute, University of Pittsburgh and University of Pittsburgh Medical Center (UPMC), Pittsburgh, PA

${ }^{3}$ Heart and Vascular Institute, University of Pittsburgh Medical Center (UPMC), Pittsburgh, PA

${ }^{4}$ Division of Pulmonary, Allergy and Critical Care Medicine, School of Medicine, University of Pittsburgh, Pittsburgh, PA

${ }^{5}$ Division of Cardiology, School of Medicine, University of Pittsburgh, Pittsburgh, PA ${ }^{6} \mathrm{McGowan}$ Institute for Regenerative Medicine, University of Pittsburgh, Pittsburgh, PA

${ }^{7}$ Department of Mechanical Engineering and Materials Science, University of Pittsburgh, Pittsburgh, PA

${ }^{8}$ Center for Ultrasound Molecular Imaging and Therapeutics, University of Pittsburgh, Pittsburgh, PA

\section{Address correspondence to:}

Marc A. Simon, MD, MSc

Associate Professor of Medicine

Division of Cardiology, Department of Medicine,

University of Pittsburgh School of Medicine

Presbyterian University Hospital C-701, 200 Lothrop St, Pittsburgh, PA

Email: simonma@upmc.edu

Tel: 412-802-3131

Fax: 412-647-0595

\section{Keywords:}

Right Ventricular Remodeling; Aging; Right Ventricular Biomechanics; Ventricular Structure; Hemodynamics 


\section{$1 \quad$ Abstract}

2 Healthy aging has been associated with alterations in pulmonary vasculature and right ventricular (RV)

3 hemodynamics, potentially leading to RV remodeling. Despite the current evidence suggesting an

4 association between aging and alterations in RV function and higher prevalence of pulmonary hypertension

5 in the elderly, limited data exist on age-related differences in RV structure and biomechanics. In this work

6 we report our preliminary findings on the effects of healthy aging on RV structure, function, and

7 biomechanical properties. Hemodynamic measurements, biaxial mechanical testing, constitutive modeling,

8 and quantitative histological analysis were employed to study two groups of Sprague-Dawley rats: control

$9 \quad$ (11 weeks) and aging (80 weeks).

10 Aging was associated with increases in $\mathrm{RV}$ peak pressures $(\approx \uparrow 17 \%, \mathrm{p}=0.017), \mathrm{RV}$ contractility $(\approx \uparrow 52 \%, \mathrm{p}=$

$110.004)$, and $\mathrm{RV}$ wall thickness $(\approx \uparrow 34 \%, \mathrm{p}=0.002)$. Longitudinal realignment of $\mathrm{RV}$ collagen $\left(16.4^{\circ}\right.$,

$12 \mathrm{p}=0.013)$ and myofibers $\left(14.6^{\circ}, \mathrm{p}=0.017\right)$ were observed with aging, accompanied by transmural

13 cardiomyocyte loss and fibrosis. A bimodal alteration in biomechanical properties was noted, resulting in

14 increased myofiber stiffness $(\approx \uparrow 158 \%, \mathrm{p}=0.0006)$ and decreased effective collagen fiber stiffness $(\approx \downarrow 67 \%$,

$15 \mathrm{p}=0.031)$.

16 Our results demonstrate the potential of healthy aging to modulate RV remodeling via increased peak

17 pressures, cardiomyocyte loss, fiber reorientation, and altered collagen/myofiber stiffness. Some

18 similarities were observed between aging-induced remodeling patterns and those of RV remodeling in

19 pressure overload. 


\section{Introduction}

Healthy aging is associated with alterations in right ventricular (RV) structure and function in subjects with no underlying cardiopulmonary disease (D’Andrea et al., 2017; Fiechter et al., 2013; Granath et al., 1964; Innelli et al., 2009; Nakou et al., 2016). Aging results in pulmonary artery (PA) remodeling (Hosoda et al., 1984; Sicard et al., 2018), increased pulmonary vascular resistance (Ehrsam et al., 1983; Granath et al., 1964) and increased PA systolic pressures (Kane et al., 2016; Lam et al., 2009), leading to RV remodeling (Anversa et al., 1990; Chouabe et al., 2004), altered contraction dynamics (Effron et al., 1987), and decreased RV systolic strains (Chia et al., 2014). Moreover, aging results in diminished RV hypertrophy in response to pressure overload (Chouabe et al., 2004; Kuroha et al., 1991). Age-related differences exist in the 1,2 and 3-year survival rates of pulmonary hypertension $(\mathrm{PH})$ patients (Hoeper et

11 al., 2013).

In recent years, biomechanical analysis techniques have been employed to better understand the

14 Kia et al., 2020) and have closely linked RV biomechanics to function (Jang et al., 2017). Despite the

15 evidence suggesting an association between aging and alterations in RV structure/function and higher 16 prevalence of $\mathrm{PH}$ in the elderly (Hoeper and Gibbs, 2014), a large body of the literature has employed 17 younger animal models and limited data exist on age-associated differences in RV biomechanics.

In this short communication we present our preliminary findings on the effects of healthy aging on

19 RV structure, function, and biomechanical properties. Our study provides insights into how healthy aging

20 modulates RV remodeling and lays the ground for future work to study the age-associated differences in

21 RV response to pressure overload.

\section{Methods}

The data acquired during this study are available from the corresponding author on reasonable 
19 ) and old $\left(\approx 80\right.$ weeks, $\left.n_{\text {Aging }}=6\right)$ age groups were studied using a multi-scale biomechanical analysis

2 framework. Figure 1 summarizes the experimental procedures and different analysis techniques used in

3 this work. All animal procedures were approved by University of Pittsburgh's IACUC (protocol\# 18113872

4 and 19126652). Details of our experimentation/analysis techniques have been extensively discussed in prior

5 studies (Hill et al., 2014; Sharifi Kia et al., 2020). Please see supplementary materials for details on our

6 methods.

Briefly, terminal invasive pressure catheterization was performed on both groups and analyzed for common metrics of RV hemodynamics. The hearts were then harvested, and measurements were acquired for the Fulton index and RV free wall (RVFW) thickness.

To characterize the effects of aging on RV biomechanical properties, multi-protocol biaxial

11 mechanical testing was performed on RVFW specimens, followed by estimation of effective fiber-ensemble

12 biomechanical properties of myofibers and collagen. Additionally, a nonlinear anisotropic constitutive model (Choi and Vito, 1990) was used for modeling the response of each specimen to generate age-specific strain energy maps of RVFW mechanical properties. specimens, to quantify the effects of aging on RV fiber architecture (collagen stained in blue, myofibers strained in red/pink). Cardiomyocyte width was then measured from the histological sections for each group. Additionally, transmural area fractions of collagen/myofibers were quantified for each section to analyze the effects of aging on RVFW composition. Furthermore, transmural orientations of collagen and myofibers were quantified using gradient-based techniques. Collagen fiber coherency was measured transmurally to analyze the effects of aging on collagen morphology. toolbox (Berens, 2009) in MATLAB (Mathworks, Natick, MA). For all other data, in case of normality and 
was used for non-homoscedastic data, while non-normal distributions were compared using Mann-Whitney U-tests. For all purposes, $\mathrm{p}<0.05$ was considered statistically significant. Statistical analyses were performed in the R software package (R Foundation for Statistical Computing, Vienna, Austria).

\section{Results}

\subsection{RV Hemodynamics and Morphology}

Healthy aging did not show an effect on heart rate (Fig. 2a; 271.5 \pm 11.7 vs. 292.3 \pm 14.1 BPM for Aging-vs.-Control; p=0.326). Aging resulted in increased RV peak pressures (Fig. 2b; $26.8 \pm 0.9$ vs. 23.0 $\pm 0.9 \mathrm{mmHg}$ for Aging-vs.-Control; $\mathrm{p}=0.017$ ), while showing a modest non-significant effect on enddiastolic pressures (Fig. 2c; $1.9 \pm 0.4$ vs. $1.3 \pm 0.1 \mathrm{mmHg}$ for Aging-vs.-Control; $\mathrm{p}=0.085$ ). Effects of aging

11 on load-dependent measures of RV contractility and relaxation are shown in Figure 2d. Aging significantly

12 increased $\frac{d p}{d t} \max (1611.7 \pm 90.5$ vs. $1063.8 \pm 101.7 \mathrm{mmHg} / \mathrm{s}$ for Aging-vs.-Control; $\mathrm{p}=0.004)$, but did not 13 demonstrate any effects on $\frac{d p}{d t} \min (-823.9 \pm 60.4$ vs. $-814.7 \pm 85.5 \mathrm{mmHg} / \mathrm{s}$ for Aging-vs.-Control; $\mathrm{p}=0.940)$.

14 Increased contractility index was observed for the aging group (Fig. 2e; $60.1 \pm 2.2$ vs. $45.8 \pm 3.51 / \mathrm{s}$ for 15 Aging-vs.-Control; $\mathrm{p}=0.012$ ), while the time constant of RV relaxation (tau) remained unchanged (Fig. 2f; $1610.7 \pm 1.6$ vs. $9.9 \pm 0.8 \mathrm{~ms}$ for Aging-vs.-Control; $\mathrm{p}=0.595)$. As shown in Table 1, healthy aging significantly 17 increased RV wall thickness $(0.89 \pm 0.06$ vs. $0.66 \pm 0.03 \mathrm{~mm}$ for Aging-vs.-Control; $\mathrm{p}=0.002)$, while not affecting the Fulton index $(0.26 \pm 0.03$ vs. $0.27 \pm 0.01 \mathrm{mg} / \mathrm{mg}$ for Aging-vs.-Control; $\mathrm{p}=0.140)$.

\subsection{RVFW Biomechanical Properties}

Aging demonstrated a bimodal effect on RVFW biaxial properties by resulting in increased circumferential and longitudinal stiffness under lower strains, while progressing to decreased biaxial stiffness at higher strains (Fig. 3a). A similar effect was observed on the effective fiber-ensemble stress-

24 strain properties of RVFW collagen and myofibers (Fig. 3b). Using a rule of mixtures approach, this translated into increased effective myofiber stiffness (Fig. 3c; $169.9 \pm 20.6$ vs. $65.8 \pm 4.7 \mathrm{kPa}$ for Aging-vs.- 
1 Control; $p=0.0006)$ and decreased effective collagen fiber stiffness (Fig. 3d; $23.9 \pm 3.8$ vs. $73.1 \pm 15.4 \mathrm{MPa}$

2 for Aging-vs.-Control; $\mathrm{p}=0.031$ ). Constitutive model parameters for each group are shown in Table 2.

3 Overall, the employed model showed an acceptable fit quality to our experimental data $\left(\mathrm{R}^{2}=0.95 \pm 0.01\right.$ and

$4 \quad 0.96 \pm 0.01$ for Aging and Control, respectively). Age-specific strain energy maps, representing the 5 combined effects of all model parameters, are demonstrated in Figure 3e-f for the low-strain and high6 strain regions.

\subsection{Quantitative Transmural Histology}

Representative histological sections are demonstrated for each group in Figure 4a . Aging resulted

10 in increased cardiomyocyte width (Fig. $4 \mathbf{b} ; 25.42 \pm 0.34$ vs. $14.94 \pm 0.64 \mu \mathrm{m}$ for Aging-vs.-Control;

$11 \mathrm{p}=0.0001$ ). Quantifying the transmural orientation of RVFW fibers revealed myofiber (Fig. 4c) and

12 collagen (Fig. 4d) reorientation towards the longitudinal direction at sub-endocardial levels. Overall,

13 myofibers showed similar orientations to collagen fibers. Aging significantly shifted the overall orientation

14 of myofibers (circular mean of transmural distributions, dotted lines in Fig. 4c) by $14.6^{\circ}$ towards the

15 longitudinal direction (Fig. 4e; $\mathrm{p}=0.017$ ). Similarly, the dominant orientation of collagen fibers was shifted

16 by $16.4^{\circ}(\mathrm{p}=0.013)$. Aging also resulted in cardiomyocyte loss and decreased myofiber area fractions at

17 both epicardium (Fig. 4f; $90.8 \pm 0.3 \%$ vs. 95.3 $\pm 0.7 \%$ for Aging-vs.-Control; p=0.004) and endocardium

18 (Fig. 4f; $82.4 \pm 1.5 \%$ vs. $95.3 \pm 1.9 \%$ for Aging-vs.-Control; $\mathrm{p}=0.007$ ), with a non-significant effect on the

19 mid-ventricular region (Fig. 4f; $91.8 \pm 1.3 \%$ vs. $95.4 \pm 0.5 \%$ for Aging-vs.-Control; $p=0.063$ ). Furthermore,

20 aging lead to RVFW fibrosis and increased collagen area fractions at epicardium (Fig. 4g; $5.3 \pm 0.4 \%$ vs.

$213.4 \pm 0.3 \%$ for Aging-vs.-Control; $p=0.015)$ and the mid-ventricular region (Fig. 4 g; $5.0 \pm 0.4 \%$ vs. $3.4 \pm 0.3 \%$

22 for Aging-vs.-Control; $\mathrm{p}=0.037$ ). Analyzing the coherency of collagen architectures revealed decreased

23 coherency at the endocardium (Fig. $4 \mathbf{h} ; 10.4 \pm 1.1 \%$ vs. $19.7 \pm 1.1 \%$ for Aging-vs.-Control; p=0.003), while

24 not showing any effects on other transmural regions. 


\section{Discussion}

We aimed to investigate the effects of healthy aging on RV remodeling. Aging resulted in 1) Increased peak pressures $(\approx \uparrow 17 \%)$ and RV contractility $(\approx \uparrow 52 \%) .2)$ Increased RVFW thickness $(\approx \uparrow 34 \%)$ with no effects on the Fulton index, indicating proportional right and left ventricular growth. 3) Longitudinal reorientation of collagen/myofibers, together with cardiomyocyte loss and RVFW fibrosis with decreased collagen fiber coherency. 4) Increased effective myofiber stiffness $(\approx \uparrow 158 \%)$ accompanied by decreased collagen fiber stiffness $(\approx \downarrow 67 \%)$.

The observed increase in RV peak pressures (Fig. 2b) are consistent with previous reports of increased PA systolic pressures and RV afterload with healthy aging (Kane et al., 2016; Lam et al., 2009). Furthermore, cardiomyocyte width (Fig. 4b) and RVFW thickness (Table 1) increased with aging, leading to increased organ-level contractility (Fig. 2d-e). This is similar to previous observations of RV

13 Kia et al., 2020). reorientation were noted in animal models of PH (Avazmohammadi et al., 2017a; Sharifi Kia et al., 2020).

17 Consistent with prior work (Anversa et al., 1990; Walker et al., 2006), cardiomyocyte loss (Fig. 4f) and RVFW fibrosis (Fig. 4g) were noted due to aging. Cardiomyocyte loss in the RVFW increases the

19 hemodynamic load on the remaining myocytes (Fajemiroye et al., 2018) and may explain the observed hypertrophy patterns (Fig. 4b). While requiring further experimentation, a potential explanation for RV

21 myocyte loss and extracellular matrix (ECM) deposition may be local RVFW ischemia due to aging 22 (Anversa et al., 1990).

Fiber reorientation (Fig. 4 c-d) and increased transmural change in fiber angles, lead to a less

24 anisotropic biaxial mechanical response for the aging group (Fig. 3a). Bimodal alterations in RVFW biaxial

25 properties were observed with aging (Fig. 3a). Compared to controls, the aging group showed higher 
1 stiffness in the low-strain region, followed by lower biaxial stiffness at higher strains. Moreover, aging lead

2 to increased effective myofiber stiffness (Fig. 3c). Increased myofiber stiffness and reduced tissue-level

3 ventricular stiffness at high strains have been previously documented in separate studies on age-related left

4 ventricular remodeling (Cappelli et al., 1984; Lieber et al., 2004). Potential underlying mechanisms of

5 increased myofiber stiffness include myocyte remodeling due to cell loss, as well as reduced titin

6 phosphorylation (Rain et al., 2013). Despite an increase in collagen area fractions (Fig. 4g), effective

7 collagen fiber stiffness decreased with aging (Fig. 3d). In this study, collagen stiffness measures represent

8 the effective response of collagen fibers, estimated from the tissue-level behavior. Therefore, while this

9 could indicate a decrease in the intrinsic stiffness of collagen fibers, it does not eliminate the possibility of

10 tissue-level structure/architecture affecting the observed behavior (Hill et al., 2014). For instance, reduced

11 collagen fiber coherency was detected at the endocardial levels (Fig $\mathbf{4 h}$ ), indicating a more sparse and

12 isotropic distribution of collagen fibers in the aging group (Clemons et al., 2018). This has the potential to

13 affect the load transfer mechanism of endocardial collagen fibers, contributing to reduced effective modulus

14 at the tissue level. Further experimentation is warranted on the mechanisms of collagen fiber remodeling

15 with aging. Candidate mechanisms for decreased effective collagen stiffness include 1) Lysyl oxidase-

16 mediated alterations in collagen cross-linking, 2) Reduced effective collagen stiffness due to myocyte loss

17 and altered biomechanics in the collagen/myofiber niche, hampering effective load transfer in the RVFW

18 continuum, 3) Altered collagen microstructure and crimp, resulting in delayed collagen recruitment.

21 experimentally measured data, indicating increased stiffness in the low-strain region, followed by decreased

22 stiffness at higher strains (Fig. 3e-f).

Despite low variability and strong statistics, the small sample size of our preliminary study remains

24 a limitation. Moreover, lack of volumetric hemodynamic data prevents analyzing the effects of aging on in

25 vivo RV structure. Additionally, we employed a phenomenological constitutive model for analyzing our

26 biomechanical data. Future work will focus on structurally-informed constitutive models of RV 
1 myocardium (Avazmohammadi et al., 2017b) to couple the histologically measured tissue architecture to

2 biaxial properties. Different batches of staining solution used for each group, resulted in different shades of

3 cardiomyocyte staining for control vs. aging (red vs. pink). However, this will have minimal effects on our

4 findings since segmentation thresholds for myofibers and collagen were individually selected for each

5 histological section.

6 In summary, our results demonstrate the potential of healthy aging to modulate RV remodeling via

7 increased peak pressures, cardiomyocyte loss, fibrosis, fiber reorientation and altered collagen/myofiber

8 stiffness. Some similarities were observed between aging-induced remodeling patterns and RV remodeling

9 in $\mathrm{PH}$.

10

11 Acknowledgments

12 This study was supported by the American Heart Association (AHA-20PRE35210429, D.S.K) and

13 the National Institutes of Health (NIH Grants 1R01AG058659, 2P01HL103455, and UL1 TR001857,

14 M.A.S; 2R01HL130261, 2R01HL113178, and R01HL150638, E.A.G). The funding sources had no

15 involvement in design of the study, data acquisition or interpretation.

16

\section{Conflict of Interest Statement}

18 D.S.K: None, Y.S: None, T.N.B: None, E.A.G: None, K.K: None, M.A.S: Research support from

19 Novartis, Aadi. Consultancy fees from Complexa, Actelion, United Therapeutics, Acceleron 


\section{References}

Anversa, P., Palackal, T., Sonnenblick, E.H., Olivetti, G., Meggs, L.G., Capasso, J.M., 1990. Myocyte cell loss and myocyte cellular hyperplasia in the hypertrophied aging rat heart. Circ. Res. 67, 871-885. doi:10.1161/01.RES.67.4.871

Avazmohammadi, R., Hill, M., Simon, M., Sacks, M., 2017a. Transmural remodeling of right ventricular myocardium in response to pulmonary arterial hypertension. APL Bioeng. 1, 016105. doi:10.1063/1.5011639

Avazmohammadi, R., Hill, M.R., Simon, M.A., Zhang, W., Sacks, M.S., 2017b. A novel constitutive model for passive right ventricular myocardium: evidence for myofiber-collagen fiber mechanical coupling. Biomech. Model. Mechanobiol. 16, 561-581. doi:10.1007/s10237-016-0837-7

Avazmohammadi, R., Mendiola, E.A., Li, D.S., Vanderslice, P., Dixon, R.A.F., Sacks, M.S., 2019. Interactions Between Structural Remodeling and Hypertrophy in the Right Ventricle in Response to Pulmonary Arterial Hypertension. J. Biomech. Eng. 141, 0910161-13. doi:10.1115/1.4044174

Berens, P., 2009. CircStat: a MATLAB toolbox for circular statistics. J. Stat. Softw. 31, 1-21. doi:10.18637/jss.v031.i10

Cappelli, V., Forni, R., Poggesi, C., Reggiani, C., Ricciardi, L., 1984. Age-dependent variations of diastolic stiffness and collagen content in rat ventricular myocardium. Arch. Physiol. Biochem. 92, 93-106. doi:10.3109/13813458409071133

Chia, E.M., Hsieh, C.H.C., Boyd, A., Pham, P., Vidaic, J., Leung, D., Thomas, L., 2014. Effects of age and gender on right ventricular systolic and diastolic function using two-dimensional speckle-tracking strain. J. Am. Soc. Echocardiogr. 27, 1079-1086.e1. doi:10.1016/j.echo.2014.06.007

Choi, H.S., Vito, R.P., 1990. Two-dimensional stress-strain relationship for canine pericardium. J. Biomech. Eng. 112, 153-159. doi:10.1115/1.2891166

Chouabe, C., Ricci, E., Amsellem, J., Blaineau, S., Dalmaz, Y., Favier, R., Pequignot, J.M., Bonvallet, R., 2004. Effects of aging on the cardiac remodeling induced by chronic high-altitude hypoxia in rat. Am. J. Physiol. - Hear. Circ. Physiol. 287, H1246-H1253. doi:10.1152/ajpheart.00199.2004

Clemons, T.D., Bradshaw, M., Toshniwal, P., Chaudhari, N., Stevenson, A.W., Lynch, J., Fear, M.W., Wood, F.M., Iyer, K.S., 2018. Coherency image analysis to quantify collagen architecture: Implications in scar assessment. RSC Adv. 8, 9661-9669. doi:10.1039/c7ra12693j

D’Andrea, A., Vriz, O., Carbone, A., Ferrara, F., Di Maio, M., Cocchia, R., Tagliamonte, G., Acri, E., Driussi, C., Pezzullo, E., Citro, R., Cittadini, A., Calabrò, R., Giovanna Russo, M., Bossone, E., 2017. The impact of age and gender on right ventricular diastolic function among healthy adults. J. Cardiol. 70, 387-395. doi:10.1016/j.jjcc.2016.12.005

Effron, M.B., Bhatnagar, G.M., Spurgeon, H.A., Ruaño-Arroyo, G., Lakatta, E.G., 1987. Changes in myosin isoenzymes, ATPase activity, and contraction duration in rat cardiac muscle with aging can be modulated by thyroxine. Circ. Res. 60, 238-245. doi:10.1161/01.RES.60.2.238

Ehrsam, R.E., Perruchoud, A., Oberholzer, M., Burkart, F., Herzog, H., 1983. Influence of age on pulmonary haemodynamics at rest and during supine exercise. Clin. Sci. 65, 653-660. 


\section{doi:10.1042/cs0650653}

Fajemiroye, J.O., Cunha, L.C. Da, Saavedra-Rodríguez, R., Rodrigues, K.L., Naves, L.M., Mourão, A.A., Silva, E.F. Da, Williams, N.E.E., Martins, J.L.R., Sousa, R.B., Rebelo, A.C.S., Reis, A.A.D.S., Santos, R.D.S., Ferreira-Neto, M.L., Pedrino, G.R., 2018. Aging-Induced Biological Changes and Cardiovascular Diseases. Biomed Res. Int. 2018, 1-14. doi:10.1155/2018/7156435

Fiechter, M., Fuchs, T.A., Gebhard, C., Stehli, J., Klaeser, B., Stähli, B.E., Manka, R., Manes, C., Tanner, F.C., Gaemperli, O., Kaufmann, P.A., 2013. Age-related normal structural and functional ventricular values in cardiac function assessed by magnetic resonance. BMC Med. Imaging 13, 1-6. doi:10.1186/1471-2342-13-6

Granath, A., Jonsson, B., Strandell, T., 1964. Circulation in Healthy Old Men, Studied by Right Heart Catheterization at Rest and During Exercise in Supine and Sitting Position. Acta Med. Scand. 176, 425-446. doi:10.1111/j.0954-6820.1964.tb00949.x

Hill, M.R., Simon, M.A., Valdez-Jasso, D., Zhang, W., Champion, H.C., Sacks, M.S., 2014. Structural and Mechanical Adaptations of Right Ventricle Free Wall Myocardium to Pressure Overload. Ann. Biomed. Eng. 42, 2451-2465. doi:10.1007/s10439-014-1096-3

Hoeper, M.M., Gibbs, J.S.R., 2014. The changing landscape of pulmonary arterial hypertension and implications for patient care. Eur. Respir. Rev. 23, 450-457. doi:10.1183/09059180.00007814

Hoeper, M.M., Huscher, D., Ghofrani, H.A., Delcroix, M., Distler, O., Schweiger, C., Grunig, E., Staehler, G., Rosenkranz, S., Halank, M., Held, M., Grohé, C., Lange, T.J., Behr, J., Klose, H., Wilkens, H., Filusch, A., Germann, M., Ewert, R., Seyfarth, H.J., Olsson, K.M., Opitz, C.F., Gaine, S.P., Vizza, C.D., Vonk-Noordegraaf, A., Kaemmerer, H., Gibbs, J.S.R., Pittrow, D., 2013. Elderly patients diagnosed with idiopathic pulmonary arterial hypertension: Results from the COMPERA registry. Int. J. Cardiol. 168, 871-880. doi:10.1016/j.ijcard.2012.10.026

Hosoda, Y., Kawano, K., Yamasawa, F., Ishii, T., Shibata, T., Inayama, S., 1984. Age-Dependent Changes of Collagen and Elastin Content in Human Aorta and Pulmonary Artery. Angiology 35, 615-621. doi:10.1177/000331978403501001

Innelli, P., Esposito, R., Olibet, M., Nistri, S., Galderisi, M., 2009. The impact of ageing on right ventricular longitudinal function in healthy subjects: A pulsed tissue Doppler study. Eur. J. Echocardiogr. 10, 491-498. doi:10.1093/ejechocard/jen313

Jang, S., Vanderpool, R.R., Avazmohammadi, R., Lapshin, E., Bachman, T.N., Sacks, M., Simon, M.A., 2017. Biomechanical and hemodynamic measures of right ventricular diastolic function: Translating tissue biomechanics to clinical relevance. J. Am. Heart Assoc. 6, 1-10. doi:10.1161/JAHA.117.006084

Kane, G.C., Sachdev, A., Villarraga, H.R., Ammash, N.M., Oh, J.K., McGoon, M.D., Pellikka, P.A., McCully, R.B., 2016. Impact of age on pulmonary artery systolic pressures at rest and with exercise. Echo Res. Pract. 3, 53-61. doi:10.1530/ERP-16-0006

Kuroha, M., Isoyama, S., Ito, N., Takishima, T., 1991. Effects of age on right ventricular hypertrophic response to pressure-Overload in rats. J. Mol. Cell. Cardiol. 23, 1177-1190. doi:10.1016/00222828(91)90206-2 
Lam, C.S.P., Borlaug, B.A., Kane, G.C., Enders, F.T., Rodeheffer, R.J., Redfield, M.M., 2009. Ageassociated increases in pulmonary artery systolic pressure in the general population. Circulation 119, 2663-2670. doi:10.1161/CIRCULATIONAHA.108.838698

Lieber, S.C., Aubry, N., Pain, J., Diaz, G., Kim, S.J., Vatner, S.F., 2004. Aging increases stiffness of cardiac myocytes measured by atomic force microscopy nanoindentation. Am. J. Physiol. - Hear. Circ. Physiol. 287, H645-H651. doi:10.1152/ajpheart.00564.2003

Nakou, E.S., Parthenakis, F.I., Kallergis, E.M., Marketou, M.E., Nakos, K.S., Vardas, P.E., 2016. Healthy aging and myocardium: A complicated process with various effects in cardiac structure and physiology. Int. J. Cardiol. 209, 167-175. doi:10.1016/j.ijcard.2016.02.039

Rain, S., Handoko, M.L., Trip, P., Gan, C.T.J., Westerhof, N., Stienen, G.J., Paulus, W.J., Ottenheijm, C.A.C., Marcus, J.T., Dorfmüller, P., Guignabert, C., Humbert, M., Macdonald, P., Dos Remedios, C., Postmus, P.E., Saripalli, C., Hidalgo, C.G., Granzier, H.L., Vonk-Noordegraaf, A., Van Der Velden, J., De Man, F.S., 2013. Right ventricular diastolic impairment in patients with pulmonary arterial hypertension. Circulation 128, 2016-2025. doi:10.1161/CIRCULATIONAHA.113.001873

Sharifi Kia, D., Benza, E., Bachman, T.N., Tushak, C., Kim, K., Simon, M.A., 2020. Angiotensin ReceptorNeprilysin Inhibition Attenuates Right Ventricular Remodeling in Pulmonary Hypertension. J. Am. Heart Assoc. 9, e015708. doi:10.1161/JAHA.119.015708

Sicard, D., Haak, A.J., Choi, K.M., Craig, A.R., Fredenburgh, L.E., Tschumperlin, D.J., 2018. Aging and anatomical variations in lung tissue stiffness. Am. J. Physiol. - Lung Cell. Mol. Physiol. 314, L946L955. doi:10.1152/ajplung.00415.2017

Walker, E.M., Nillas, M.S., Mangiarua, E.I., Cansino, S., Morrison, R.G., Perdue, R.R., Triest, W.E., Wright, G.L., Studeny, M., Wehner, P., Rice, K.M., Blough, E.R., 2006. Age-associated changes in hearts of male fischer 344/Brown Norway F1 rats. Ann. Clin. Lab. Sci. 36, 427-438. 
Table 1: Effects of healthy aging on RV hypertrophy (RVFW thickness) and Fulton index

\begin{tabular}{c|c|c} 
& $\begin{array}{c}\text { RVFW Thickness } \\
(\mathbf{m m})\end{array}$ & $\begin{array}{c}\text { Fulton Index } \\
\mathbf{( m g / m g )}\end{array}$ \\
\hline $\begin{array}{c}\text { Control } \\
(\mathbf{n = 9 )}\end{array}$ & $0.66 \pm 0.03$ & $0.27 \pm 0.01$ \\
\hline $\begin{array}{c}\text { Aging } \\
(\mathbf{n}=\mathbf{6})\end{array}$ & $0.89 \pm 0.06$ & $0.26 \pm 0.03$ \\
\hline p Value & 0.002 & 0.140
\end{tabular}

Table 2: Constitutive model parameters representing the circumferential $\left(b_{1}\right)$, longitudinal $\left(b_{2}\right)$ and inplane coupling $\left(b_{3}\right)$ stiffness of RV myocardium for the control and aging groups

\begin{tabular}{c|c|c|c|c} 
& \multicolumn{4}{|c}{ Model Parameters } \\
& $\mathbf{B}_{\mathbf{0}}$ & $\mathbf{b}_{\mathbf{1}} \mathbf{( k P a )}$ & $\mathbf{b}_{\mathbf{2}}(\mathbf{k P a})$ & $\mathbf{b}_{3}(\mathbf{k P a})$ \\
\hline $\begin{array}{c}\text { Control } \\
\mathbf{( n = 6 )}\end{array}$ & $0.173 \pm 0.023$ & $98.23 \pm 14.52$ & $76.33 \pm 7.53$ & $66.72 \pm 6.64$ \\
\hline $\begin{array}{c}\text { Aging } \\
\mathbf{( n = 6 )}\end{array}$ & $0.369 \pm 0.082$ & $56.01 \pm 7.12$ & $47.35 \pm 6.88$ & $43.21 \pm 3.80$ \\
\hline $\mathrm{p}$ Value & 0.044 & 0.026 & 0.017 & 0.012
\end{tabular}


Figure 1: The framework used to study RV remodeling with healthy aging. In vivo terminal invasive hemodynamic measurements were performed on young controls and aging Sprague-Dawley rats, followed by harvesting the heart and morphological measurements, biaxial mechanical testing, constitutive modeling, and quantitative transmural histological analysis.

RV: Right ventricle; OT: Outflow tract; Circ: Circumferential; Long.: Longitudinal

Figure 2: Hemodynamic measures of the effects of healthy aging on RV (a) Heart rate, (b) Peak pressure, (c) End-diastolic pressure, (d) $\frac{d p}{d t} \max$ (positive side) and $\frac{d p}{d t} \min$ (negative side), (e) Contractility index and (f) Preload-independent measure of relaxation (tau). Healthy aging significantly increased RV peak pressures and the load-dependent measures of RV contractility ( $\frac{d p}{d t} \max$ and contractility index $)$, while not affecting the heart rate, end-diastolic pressures and relaxation function $\left(\frac{d p}{d t} \min\right.$ and tau). Error bars represent standard error of the mean (SEM). * indicates $\mathrm{p}<0.05$.

RV: Right ventricle; BPM: Beats per minute; EDP: End-diastolic pressure; $\frac{d p}{d t} \max$ and min: Loaddependent measures of RV contractility and relaxation; n.s: Non-significant.

Figure 3: Effects of healthy aging on (a) Biaxial mechanical properties of RV myocardium, (b) Effective fiber-ensemble mechanical properties of combined myofiber-collagen bundles, (c) Effective myofiber stiffness, (d) Effective collagen fiber stiffness, (e) Strain energy maps of RVFW in the low-strain region (circumferential-longitudinal strain space) and (f) Strain energy maps of RVFW in the high-strain region. Healthy aging modulates the biomechanical properties of RVFW in a bimodal manner by increasing the effective myofiber stiffness while decreasing collagen fiber stiffness. Strain energy maps demonstrate increased RVFW stiffness in the low-strain region with healthy aging (mostly dominated by myofibers), followed by decreased RVFW stiffness in the high-strain region post-collagen recruitment. Error bars represent standard error of the mean $(\mathrm{SEM})$. indicates $\mathrm{p}<0.05$. 
RV: Right ventricle; RVFW: Right ventricular free wall; $2^{\text {nd }}$ P-K Stress: $2^{\text {nd }}$ Piola-Kirchhoff stress; Circ.: Circumferential; Long.: Longitudinal; EFE $2^{\text {nd }}$ P-K Stress: Effective fiber-ensemble $2^{\text {nd }}$ Piola-Kirchhoff stress.

Figure 4: Histological analysis of the effects of healthy aging on RV structure. (a) Representative transmural histological sections of the RVFW (Red/Pink: Myofiber, Blue: Collagen) and effects of aging on (b) Cardiomyocyte hypertrophy (myocyte width), (c) Transmural myofiber orientations, (d) Transmural collagen fiber orientations, (e) Dominant myofiber orientations, (f) Transmural myofiber content (area fraction), (g) Transmural collagen content (area fraction) and (h) Transmural collagen fiber coherency. Healthy aging results in cardiomyocyte hypertrophy, in addition to reorientation of sub-endocardial collagen and myofibers towards the longitudinal direction. This is accompanied by loss of cardiomyocytes, RVFW fibrosis and decreased collagen fiber coherency at endocardium. Error bars represent standard error of the mean (SEM). * indicates $\mathrm{p}<0.05$.

RV: Right ventricle; RVFW: Right ventricular free wall; Epi: Epicardium; Mid: Mid-ventricular region; Endo: Endocardium. 


\section{Figure 1:}

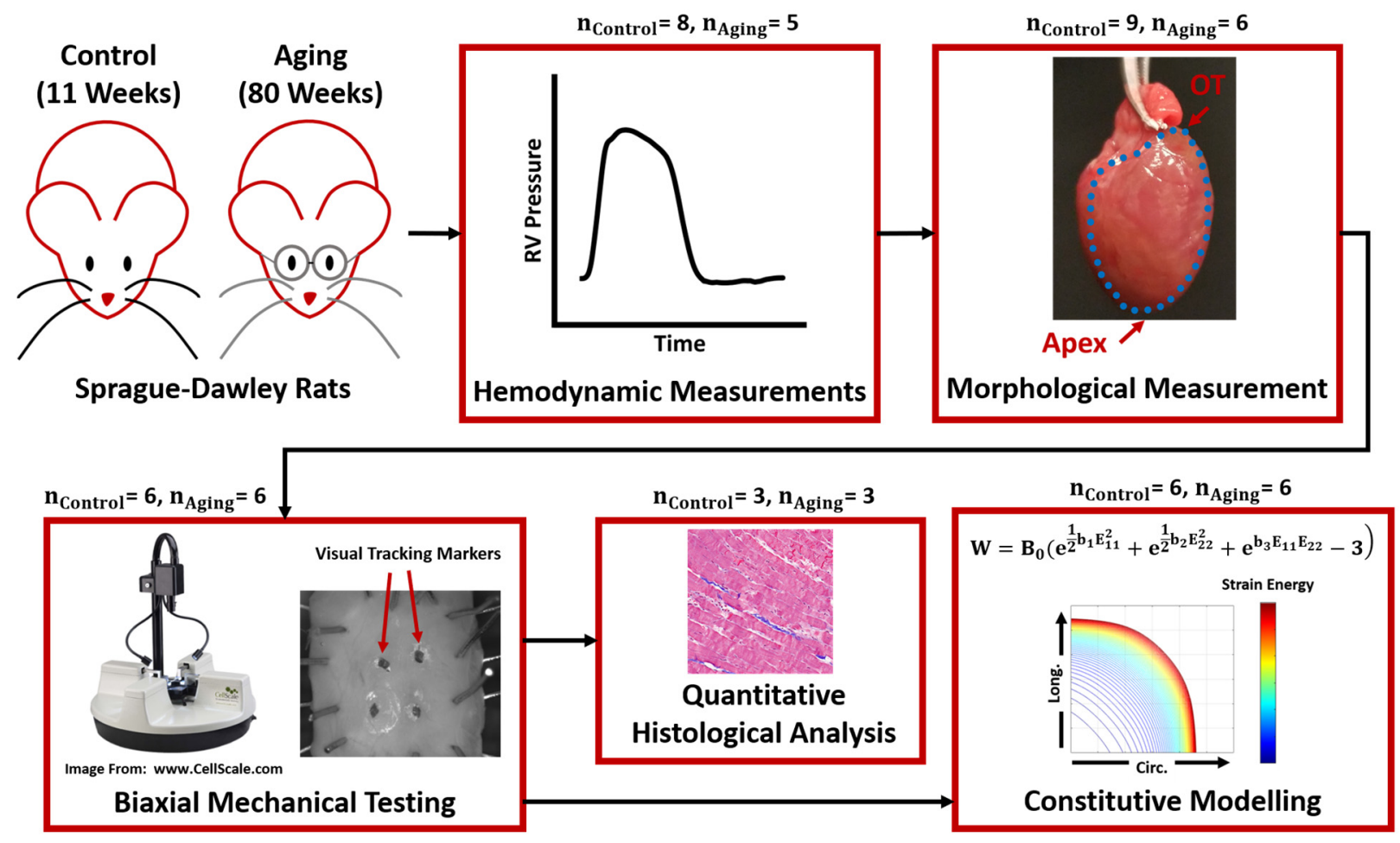




\section{Figure 2:}

a
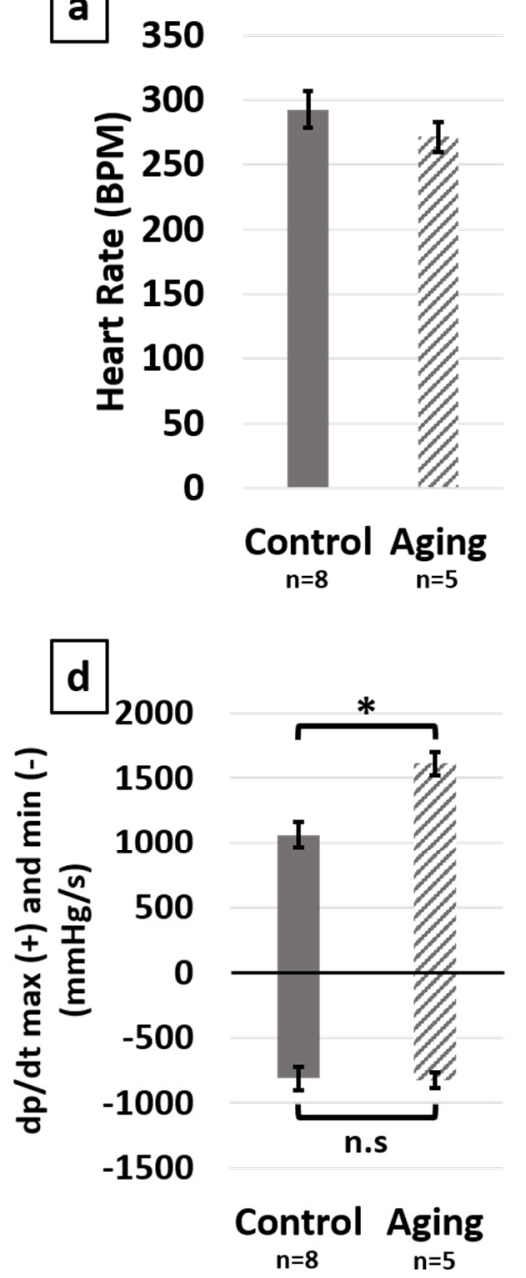

b

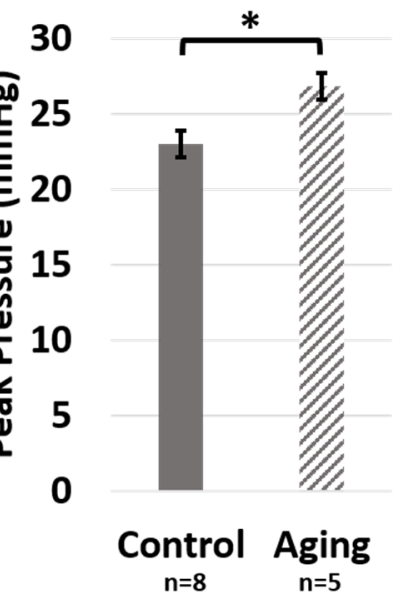

e

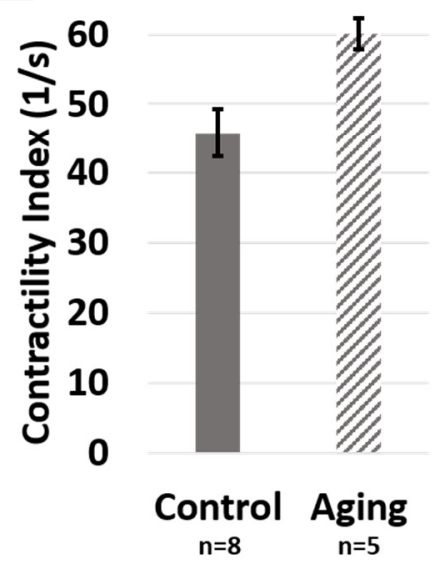

c

2.5

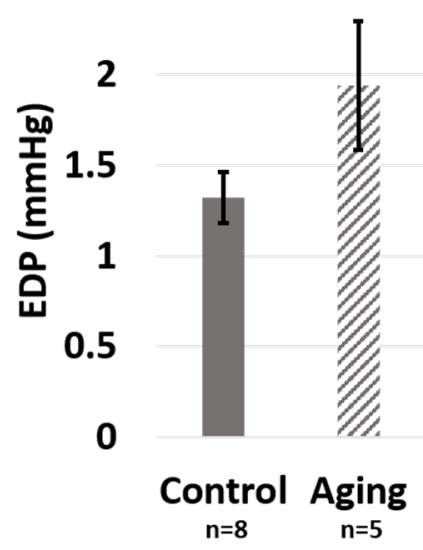

f 


\section{Figure 3:}

a

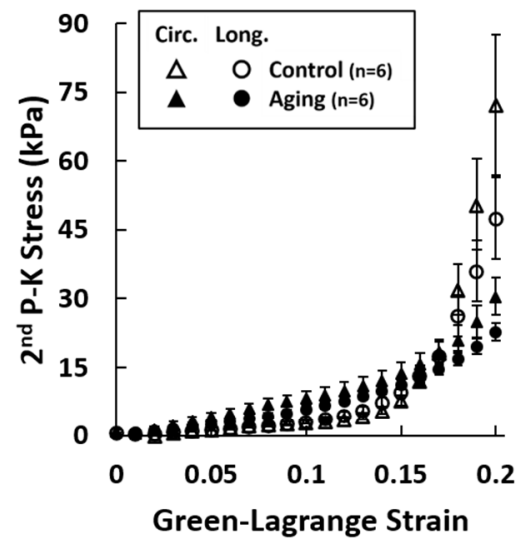

c

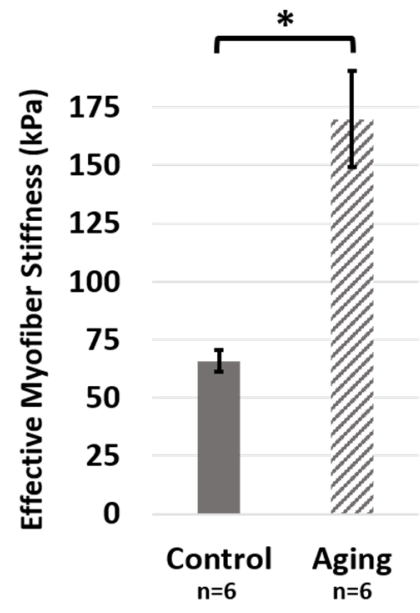

e

Low-Strain

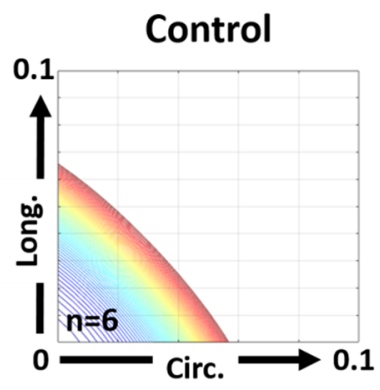

f

High-Strain

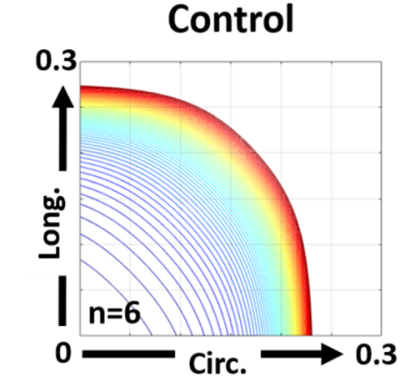

b

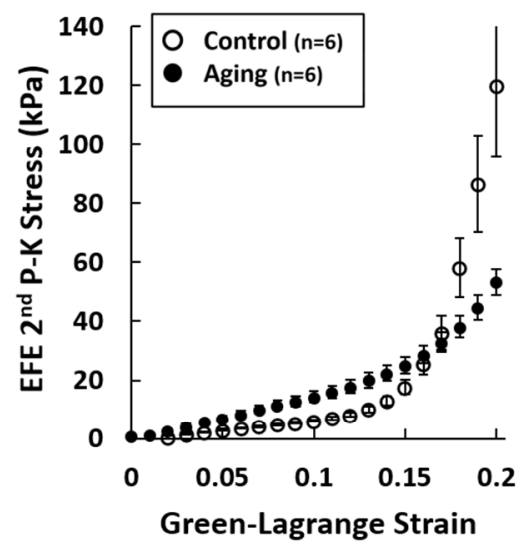

d
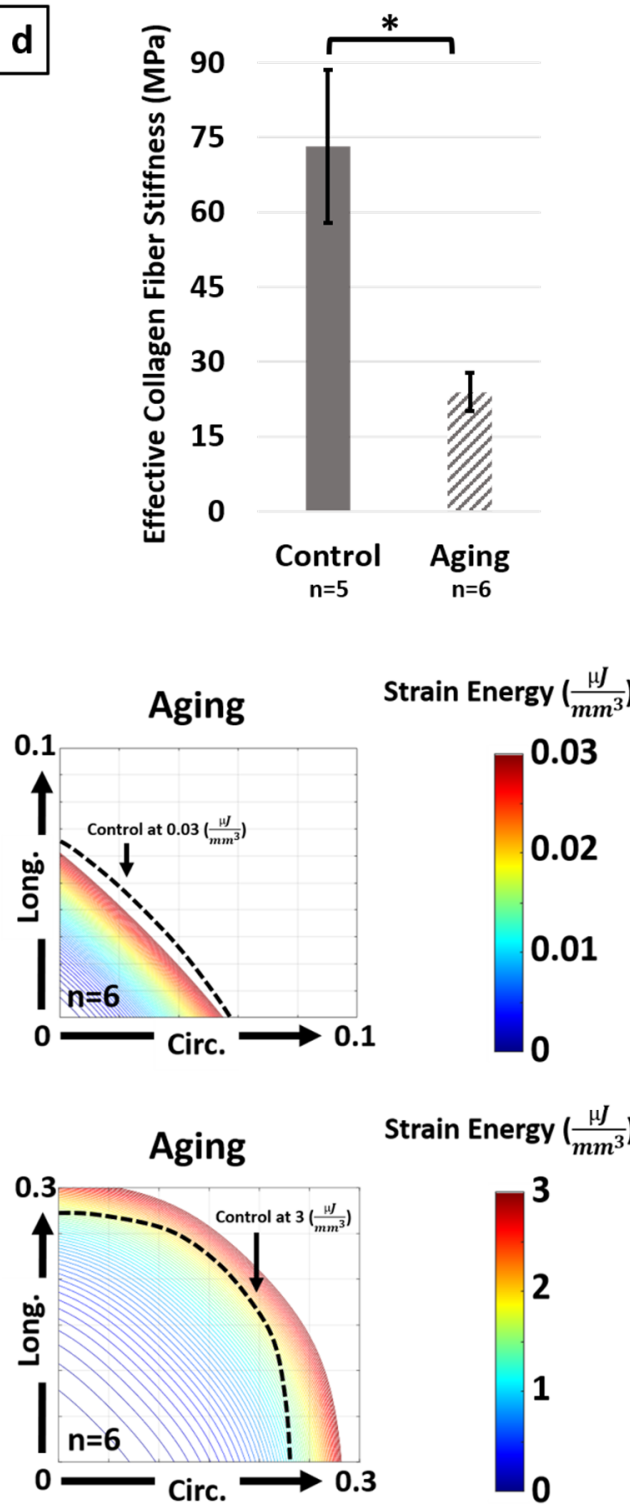


\section{Figure 4:}
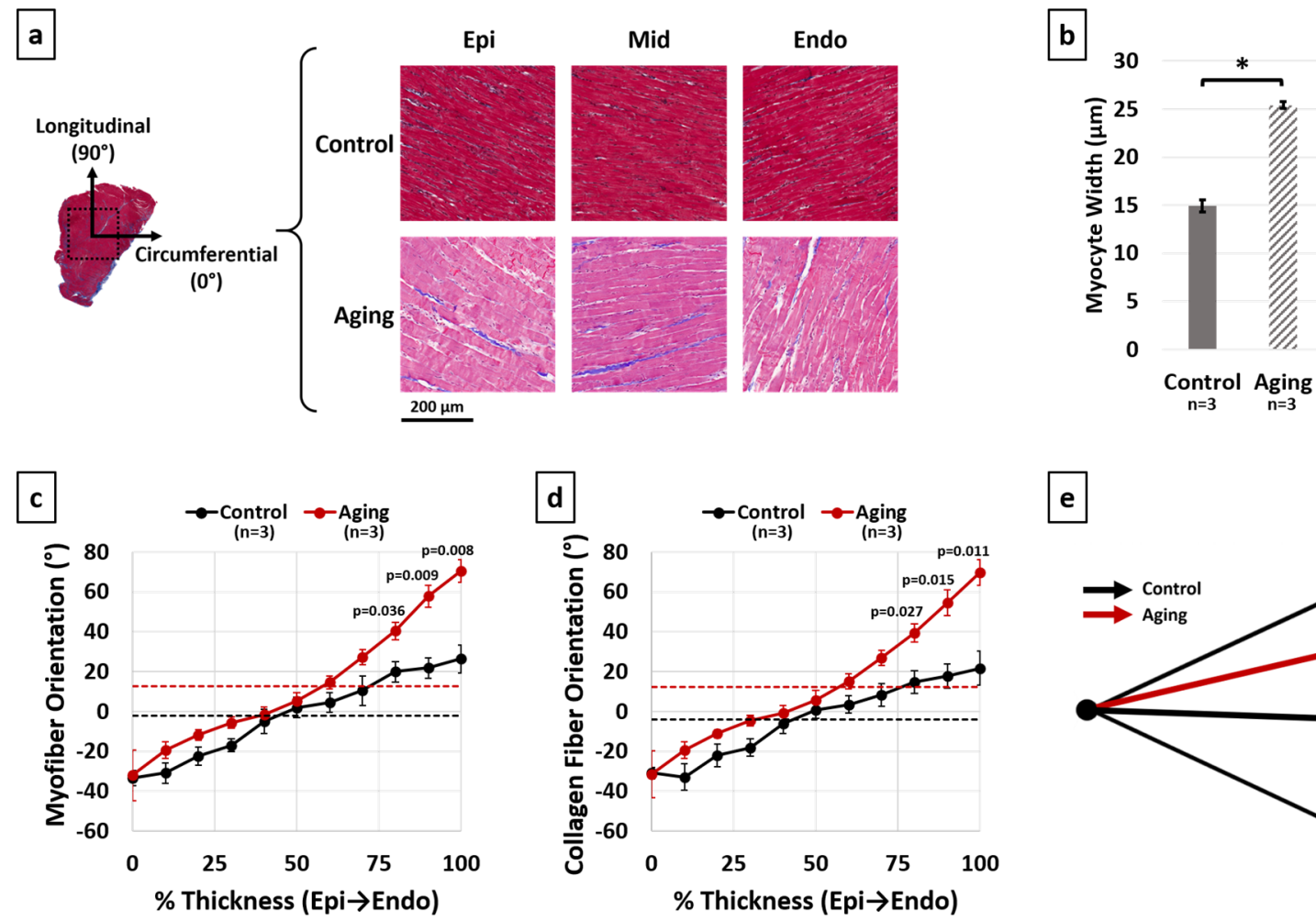

e
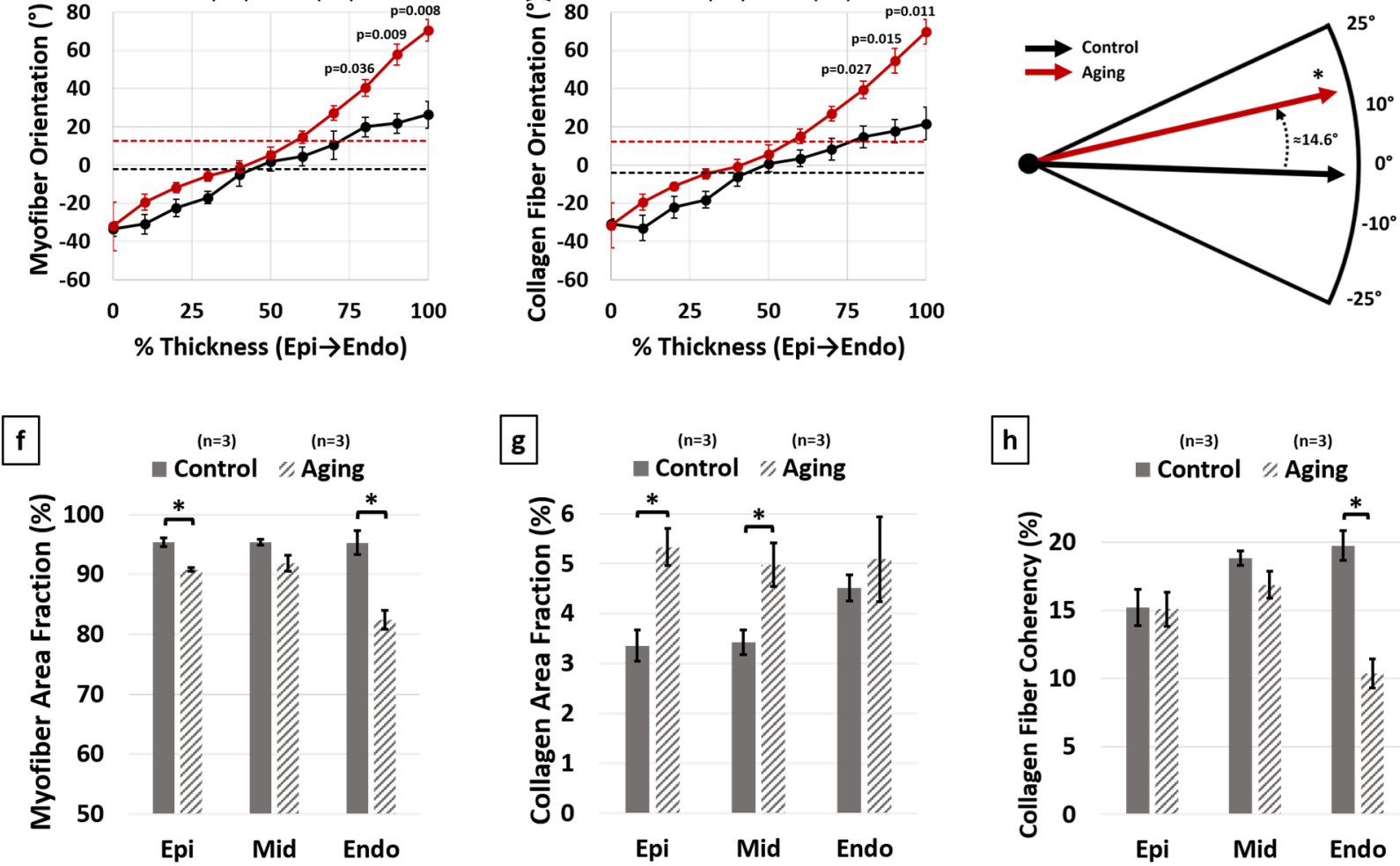